\title{
Hot topic: Bisphenol A in cow milk and dietary exposure at the farm level
}

\author{
Serena Santonicola, ${ }^{1}$ Maria Carmela Ferrante, ${ }^{1}$ Nicoletta Murru, ${ }^{1}$ Pasquale Gallo, ${ }^{2}$ \\ and Raffaelina Mercogliano ${ }^{1 *}$ \\ ${ }^{1}$ Department of Veterinary Medicine and Animal Production, University of Naples, 80137 Naples, Italy \\ ${ }^{2}$ Istituto Zooprofilattico Sperimentale del Mezzogiorno, 80055 Portici, Italy
}

\section{ABSTRACT}

Chemical hazards may enter the milk chain during primary production. The study, for the first time, investigated the occurrence of bisphenol A (BPA) levels in cow milk samples collected on the farm following manual or mechanical milking and from the cooling tank. We applied a new monitoring model based on the identification of the hazards at each stage of the milk chain to identify potential pathways for contamination along the milk chain. We evaluated exposure to BPA through milk consumption based on detected contamination levels and the temporary tolerable daily intake established by the European Food Safety Authority (EFSA). Milk samples $(\mathrm{n}=72)$ were analyzed using liquid chromatography with fluorescence detection. The mean BPA concentrations were $0.757 \mu \mathrm{g} / \mathrm{L}$ in manually milked samples, $0.580 \mu \mathrm{g} / \mathrm{L}$ in mechanically milked samples, and $0.797 \mu \mathrm{g} / \mathrm{L}$ in milk from the cooling tank. Bisphenol A occurred in the milk chain as a result of different stages of milking, and reached the highest levels at the end of the milk chain. Although the dietary intake of BPA was below the EFSA's temporary tolerable daily intake, exposure to BPA, even at low doses, through milk consumption represents a public health concern. Therefore, to ensure milk safety, new monitoring plans should be applied based on the identification of hazards at each stage of the milk chain. Key words: milk chain, bisphenol A, dietary exposure

\section{Hot Topic}

Chemical hazards may enter the milk chain at primary production, through ingestion of contaminated feed, via the uptake of chemical compounds as a result of cows grazing on contaminated soil, or via the administration of veterinary medicines to cows. Such hazards

Received July 6, 2018.

Accepted October 31, 2018.

*Corresponding author: raffaella.mercogliano@unina.it may persist at the milk collection and preparation processes of dairy products (Santonicola et al., 2017; van Asselt et al., 2017). Bisphenol A [BPA; 2,2-bis(4hydroxyphenyl) propane] is an organic compound readily biodegraded under aerobic conditions in soil and sediments. As industrial chemical, it is widely used in the production of polycarbonate plastics (used in food contact materials), epoxy resins (as internal coating in canned food), and as an antioxidant in polyvinyl chloride (PVC) materials intended to come into contact with food (EFSA, 2015). Under normal conditions of use, BPA tends to leach from food containers and may be detected in human biological matrices. Because BPA is an endocrine disruptor (i.e., it interferes with the endocrine system by mimicking blocking and triggering actions of hormones), it is assumed to have toxic effects on metabolism, growth, sexual development, stress response, insulin production, gender behavior, reproduction, and fetal development of the living body (Cirillo et al., 2015). It is also considered a contributing factor in the onset of metabolic disorders, including diabetes and obesity, and immune dysfunction (Bansal et al., 2018).

Data on BPA exposure confirm that, of all sources and across all population groups, the diet is the most important source (EFSA, 2015). Upon dietary exposure, the first-pass metabolism of BPA occurs in the wall of the gut and liver, driven by uridine diphosphate glucuronosyltransferases, with the formation of BPA mono- and diglucuronide and BPA mono- and disulfate conjugates, which are the 4 most common BPA phase II metabolites in mammalian model (Nachman et al., 2014; Ho et al., 2017). These highly water-soluble conjugates are mainly excreted in urine (Ho et al., 2017). However, BPA is able to induce or inhibit metabolizing enzymes at doses compatible with human exposure (Quesnot et al., 2014). As the diet is the main source of exposure in all population groups, the European Food Safety Authority recommends a temporary tolerable daily intake (t-TDI) of $4 \mu \mathrm{g} / \mathrm{kg}$ of BW per day for adults and children (EFSA, 2015). Currently, Regulation (EU) No 2018/213 (European Union, 2018) speci- 
fies a new specific migration limit of $0.05 \mathrm{mg} / \mathrm{kg}$ into or onto food from varnishes or coatings applied to food contact materials, and the use of BPA in the fabrication of baby bottles is prohibited.

Dairy production follows several stages from animal feed and raw milk production on the farm to further processing at a dairy company or on the farm itself (van Asselt et al., 2017). Milk products are widely consumed by infants, children, and adults, and the occurrence of quantifiable levels of BPA is a matter of public health concern. Environmental pollution may represent a contamination source of the milk chain because BPA residues are stored in adipose tissue, secreted in cow milk fat, and accumulated in fat dairy products (Georgescu and Georgescu, 2013; Grumetto et al., 2013). On the farm, BPA may be introduced during milking from plastic parts (plastic resins, PVC tubing) of milking machines or during transfer from bulk milk to storage tanks (Chege and Ndungu, 2016). Thus, BPA residues may be concentrated in the final product, reaching high contamination levels by the end of milk processing (van Asselt et al., 2017). At the dairy company, the migration of BPA from packaging materials into cow milk or dairy products could be a further cause of contamination (Galloway, 2015). Even though many studies have reported the occurrence of BPA in milk for human consumption, data on the levels and contamination pathways along the dairy chain are scarce (EFSA, 2015; Mercogliano and Santonicola, 2018).

The aims of the study were to (1) evaluate the occurrence of nonconjugated BPA levels along the milk chain at the farm; (2) apply a new monitoring model based on the identification of hazards at each stage of the milk chain to identify the potential contamination pathways for milk contamination at primary production; and (3) evaluate the potential dietary exposure considering the hypothetical consumption of raw milk collected on the farm.

A BPA standard (minimum purity of $99 \%$ ) was purchased from Sigma-Aldrich (Poole, UK). Methanol (for HPLC), acetonitrile (for HPLC), and water (for HPLC) were provided by Carlo Erba (Milan, Italy). The solidphase extraction (SPE) cartridge (Chromabond C18, Macherey-Nagel, Duran, Germany) was purchased from Delchimica (Naples, Italy). A stock solution $(0.1 \mathrm{mg} /$ $\mathrm{mL}$ ) of BPA was prepared in acetonitrile and stored at $4^{\circ} \mathrm{C}$. Analyses were carried out using a Jasco HPLC apparatus equipped with a Jasco quaternary pump 2089 plus, combined with a Jasco fluorescence detector 821-Fp (HPLC with fluorescence detection, HPLC/ FD, Jasco Inc., Easton, MD). A Synergi column $4 \mu \mathrm{m}$ Fusion-RP $80 \AA(250 \mathrm{~mm}$ length $\times 4.60 \mathrm{~mm}$ inside diameter; Phenomenex, Torrance, CA) was used.
One hundred thirty-two Brown Swiss (average 5 yr old; producing milk with fat content of $4.5 \%$ ) was selected from a cattle farm in Campania (Italy). The basic feed for the milking cows consisted of $45 \%$ dry and green fodder and $55 \%$ grass and maize silage. At the farm, milking occurred mechanically twice a day in the stables, once in the morning and once in the evening. The milking system consisted of rubber tubes, stainless steel pipes, and milking clusters of rubber and stainless steel. The milk receiver was made from hard plastic, stainless steel, and rubber, and the cooling tank was made of stainless steel with a few rubber sealants. Milk was extracted using a vacuum and collected in the cooling tank. Every 2 to $3 \mathrm{~d}$, the cooling tank was emptied by a milk collecting company that delivered the milk to the dairy factory. The experimental design considered each phase of milk production. A total of 4 feed samples and 72 milk samples were collected. One feed sample, consisting of fodder and silage, was collected monthly for 4 mo. The milk samples from the morning milking were collected twice weekly for 12 wk with 3 types of samples: (1) milk obtained by hand before the mechanical milking process, (2) milk from machine milking, and (3) milk collected from the cooling tank. The manually and mechanically collected samples were derived from a pool of 20 cows, whereas the sample from the cooling tank was a pooled sample from all cows present on the farm. After sampling, milk was refrigerated in glass containers at $\pm 4^{\circ} \mathrm{C}$ until the time of analysis. All necessary precautions were taken to avoid contamination with BPA during sample preparation (Watabe et al., 2004; Ye et al., 2013). The use of plastic materials was avoided during sample collection, preparation, and analysis and, when possible, we introduced additional washing steps for contact materials. All glass materials, including collection flasks, were rinsed before collection with dichloromethane. Water was filtered through a Millipore purification system (Millipore, Billerica, MA) in which new cartridges and UV lamp were installed before sample analysis. The concentration of BPA in the organic solvents was found to be below the limit of detection. Preparation of BPA stock solutions was performed in a room separate from the analytical laboratories to prevent cross-contamination. For BPA determination in feed, a sample of $10.0 \mathrm{~g}$ of fodder and 5.0 $\mathrm{g}$ of silage was homogenized and purified according to the method of Maradonna et al. (2014). Feed samples were purified by Affinimip SPE Bisphenols cartridges (Polyntell SA, Paris, France), based on molecularly imprinted polymers (MIP). After clean up, $5 \mu \mathrm{L}$ of feed extract was analyzed. The quantitative analysis of BPA was performed using an HPLC coupled to tandem MS (liquid chromatography electrospray ionization QTrap 
MS/MS) system, equipped with an Agilent 1200 Series HPLC (ABSciex, Concord, ON, Canada), consisting of a binary pump and an autosampler with temperature control, and a mass spectrometer QTrap 4000 with a TurboIon Spray source (ABSciex). In all the analyzed samples, a deuterated isotope of the compound (BPAd16; Sigma-Aldrich, Sheboygan Falls, WI) was introduced. For analysis of samples, blank samples spiked with BPA at $50 \mu \mathrm{g} / \mathrm{kg}$ were prepared during each working session for quality control. Reagent blanks, calibration curve standards, and quality control samples were analyzed during all working sessions. All analyses were performed in duplicate, and the mean values of positive samples were reported. For BPA quantification in feed samples, the calculations were carried out by using labeled BPA-d16 as the internal standard. Bisphenol A was separated on a $2.6-\mu \mathrm{m}$ particle, $100 \mathrm{~mm} \times 4.6 \mathrm{~mm}$ Kinetex PFP stainless steel column (Phenomenex) at $25^{\circ} \mathrm{C}$, run at a flow rate of $0.50 \mathrm{~mL} / \mathrm{min}$, with a linear gradient elution. Tandem mass spectrometry (MS/MS) analyses were performed in multiple reaction monitoring mode and negative ionization (-MRM). For both BPA and BPA-d16, the most abundant product ions were selected as quantifier ions (Q); for BPA, a qualifier ion (q) was also selected for unambiguous confirmation. Identification of BPA was based on the retention time of both quantifier and qualifier product ions. The peak area ratios of BPA and BPA-d16 were compared with BPA standard concentrations, and calibration curves were calculated by linear regression. Concentrations of BPA in feed samples and quality control samples were calculated by interpolation of the calibration curves. Method trueness, in terms of mean recovery, was calculated via external calibration standard curves. The limit of detection (LOD) of the method was $0.005 \mu \mathrm{g} /$ $\mathrm{kg}$, and the limit of quantification (LOQ) was $0.016 \mu \mathrm{g} /$ $\mathrm{kg}$. The milk samples were prepared by adding $2.5 \mathrm{~mL}$ of milk to $7.5 \mathrm{~mL}$ of deionized water and sonicating for 30 min at room temperature in an ultrasonic apparatus (40 kHz; Branson Ultrasonic 2210, Branson Ultrasonics, Danbury, CT). Samples were then loaded into an SPE cartridge previously conditioned with $10.0 \mathrm{~mL}$ of acetonitrile and equilibrated with $10.0 \mathrm{~mL}$ of deionized water. The loaded SPE cartridge was washed with 20.0 $\mathrm{mL}$ of water and with 2 different solutions of water and methanol (80:20 and 60:40, vol/vol) under vacuum. The analytes were eluted with $10.0 \mathrm{~mL}$ of acetonitrile. Only glassware was used throughout the analyses to avoid any possible release of BPA from plastic equipment. Quantitative analysis was performed by HPLC/ FD, equipped with a $20-\mu \mathrm{L}$ loop. The mobile phase was acetonitrile:water $(70: 30, \mathrm{vol} / \mathrm{vol})$. The analyses were carried out at room temperature at a flow rate of 0.9
$\mathrm{mL} / \mathrm{min}$ in isocratic mode. The fluorescence detector was set at excitation and emission wavelengths of 273 and $300 \mathrm{~nm}$, respectively; BPA was identified based on retention time and quantification was performed using an external standard method.

Quality parameters of linearity, LOD, LOQ, precision, accuracy, and selectivity were evaluated to validate the method. Milk samples previously determined to be BPA-free were used as blanks to verify method selectivity. After spiking with known concentrations of BPA, these samples were used to confirm the assignment of peak identity and to calculate the linearity, precision, and accuracy of the method. Quantitative analysis of BPA was performed based on the calibration curve obtained by analysis of blank samples spiked with $\mathrm{BPA}$ to obtain concentrations in a range of 0.03 to $100 \mu \mathrm{g} / \mathrm{L}$. An external standard procedure was used for calibration (Figure 1). The correlation coefficient (r) between the peak area and BPA was 0.9969. The LOD and LOQ were 0.01 and $0.03 \mu \mathrm{g} / \mathrm{kg}$, respectively. Precision was expressed as both repeatability (intraday precision) and intermediate precision (interday precision) at 3 concentration levels $(1.0,10.0$, and $50.0 \mu \mathrm{g} / \mathrm{L}$ ). Intraday and interday precision values were based on 5 and 10 measurements, respectively, and the relative standard deviation did not exceed $5 \%$ for intraday and $10 \%$ for interday precision. Accuracy of the method was evaluated by recovery of the tested BPA in spiked blank samples, treated by the sample preparation procedure described, at 3 concentration levels $(1.0,10.0$, and $50.0 \mu \mathrm{g} / \mathrm{L}$ ). Recovery values ranged from 70 to $100 \%$. To characterize the background contamination, laboratory blanks and spiked matrices were analyzed to check for instrumental drift, matrix interference, and contamination of solvent and glassware during analysis of milk samples in every batch.

For evaluation of BPA exposure, we considered the hypothetical consumption of milk collected on the farm. To calculate BPA dietary intake, based on the recommended t-TDI, we used data on BW, age (Beghini, 1981), and daily milk and cheese consumption of Italian consumers (Leclercq et al., 2009; Table 1). Milk categories consisted of milk; milk products, including liquid, condensed, and powder forms; infant formula; human milk; milk substitutes (e.g., soy, rice, oat); and flavored milks (e.g., packaged chocolate-flavored milk). To enhance our overview of potential consumer intake of BPA, consumption of cheese and cheese-based products was also considered. Daily intake through cheese-based products was calculated based on the assumption that 1 $\mathrm{kg}$ of cheese requires about $5 \mathrm{~L}$ of milk for manufacture. Cheese categories consisted of different cheeses (e.g., mozzarella, Parmesan, Edam, feta, Fontina), flavored 


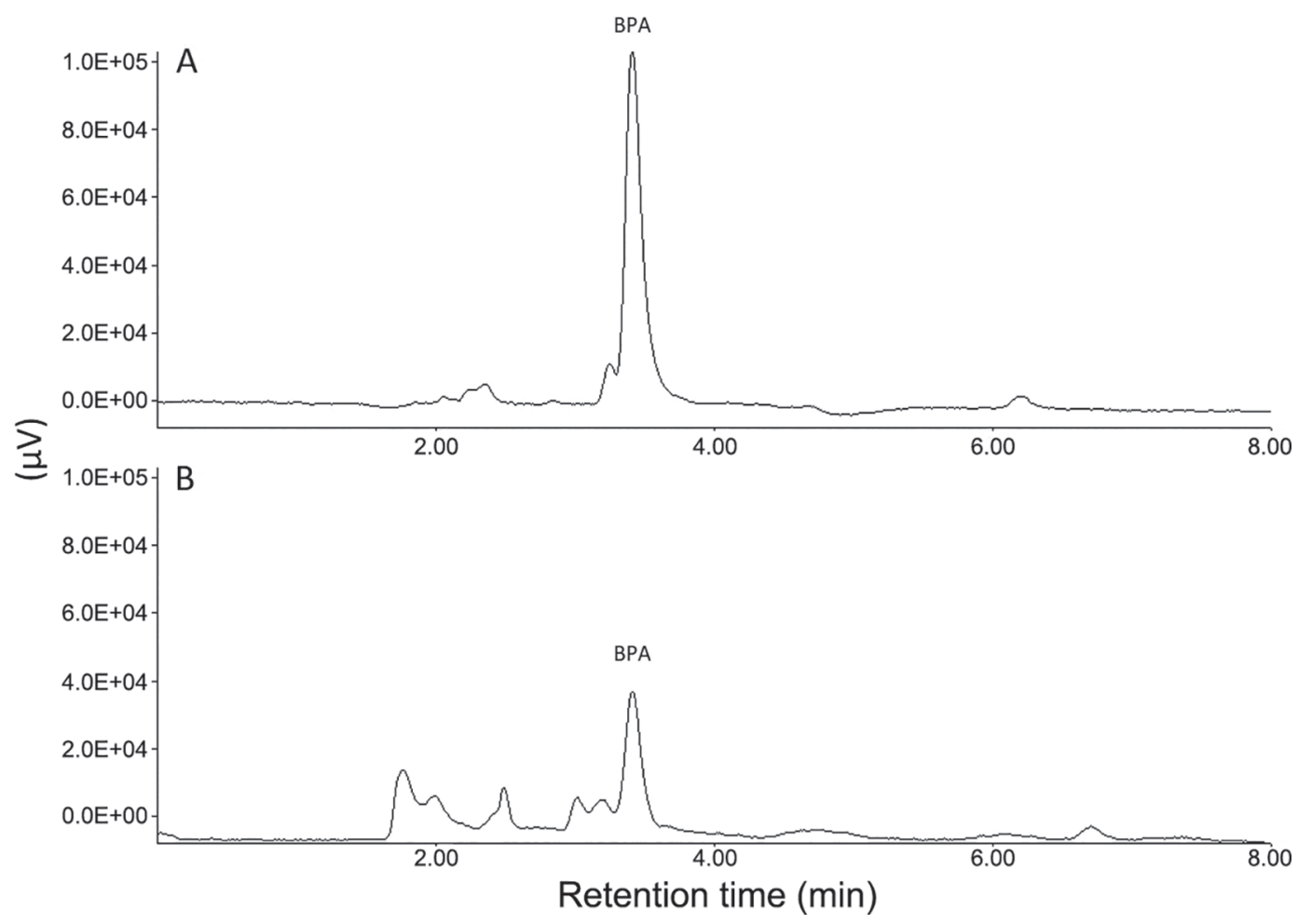

Figure 1. Liquid chromatography with fluorescence detection (LC-FD) chromatograms of a $1 \mu \mathrm{g} / \mathrm{L}$ standard solution of bisphenol A (BPA; A) and a milk sample (B). The BPA peak is shown.

sweet cheese for children, cheese substitutes (e.g., tofu), and homogenized cheese products for infants (Beghini, 1981; Leclercq et al., 2009). To measure BPA daily intake, we applied the following formula:

$$
\text { Daily intake }=\frac{C \times d I R}{B W}
$$

where $C$ is the mean BPA concentration $(\mu \mathrm{g} / \mathrm{kg}$ of lipid weight) found across sample units of analyzed cow milk; $d I R$ is the daily ingestion rate (i.e., daily consumption of milk in Italy); and BW is the corresponding average BW of consumers. Daily intakes $(\mu \mathrm{g} / \mathrm{kg}$ of BW per day) were calculated based on the average milk consumption and average BPA concentration measured in the samples collected manually, mechanically, and from the cooling tank.

No quantifiable BPA concentrations were detected in animal feed samples, whereas BPA levels in milk samples ranged from 0.035 to $2.776 \mu \mathrm{g} / \mathrm{L}$. The mean concentrations averaged $0.757 \mu \mathrm{g} / \mathrm{L}$ in manually obtained milk, $0.580 \mu \mathrm{g} / \mathrm{L}$ in mechanically milked samples, and $0.797 \mu \mathrm{g} / \mathrm{L}$ in milk from the cooling tank (Table 2 ). The results for dietary intake ranged from 0.0011 $\mu \mathrm{g} / \mathrm{kg}$ of BW per day (mechanically obtained milk, in consumers $18-65$ yr of age) to $0.022 \mu \mathrm{g} / \mathrm{kg}$ of BW per

Table 1. Mean daily consumption (g/d) of milk and cheese in infants (1-3 yr), children (3-10 yr), teenagers (10-18 yr), adults (18-65 yr), and elderly (>65 yr) male and female consumers (data from Beghini, 1981; Leclercq et al., 2009)

\begin{tabular}{|c|c|c|c|c|c|}
\hline \multirow[b]{2}{*}{ Age (yr) } & \multirow{2}{*}{$\begin{array}{l}\text { Weight } \\
(\mathrm{kg})\end{array}$} & \multicolumn{2}{|c|}{ Milk consumption (g/d) } & \multicolumn{2}{|c|}{ Cheese consumption $(\mathrm{g} / \mathrm{d})$} \\
\hline & & Mean & $\mathrm{SD}$ & Mean & $\mathrm{SD}$ \\
\hline $1-3$ & $10-14$ & 340.6 & 256.4 & 28.9 & 22.0 \\
\hline $3-10$ & $15-33$ & 205.7 & 110.3 & 45.7 & 34.2 \\
\hline $10-18$ & $34-60$ & 180.4 & 107.8 & 61.2 & 40.3 \\
\hline $18-65$ & $61-80$ & 135.3 & 97.2 & 61.8 & 42.4 \\
\hline$>65$ & $61-80$ & 157.3 & 98.2 & 57.7 & 38.7 \\
\hline
\end{tabular}


Table 2. Concentrations of bisphenol A in cow milk milked by hand or machine, in raw milk from the cooling tank, and in feed samples

\begin{tabular}{lcccc}
\hline & & \multicolumn{3}{c}{ Bisphenol A $(\mu \mathrm{g} / \mathrm{L})$} \\
\cline { 3 - 5 } Sample & Nomples & Range & Median & Mean \\
\cline { 3 - 5 } & & $0.081-2.492$ & 0.546 & 0.757 \\
Milked by hand & 24 & $0.089-1.835$ & 0.416 & 0.580 \\
Milked by machine & 24 & $0.035-2.776$ & 0.467 & 0.797 \\
Cooling tank & 24 & ND $^{1}$ & ND & ND \\
Feed samples & 4 & &
\end{tabular}

${ }^{1}$ Not detected (concentrations below the limit of detection).

day (milk from the cooling tank, in consumers $1-3$ yr of age; Table 3$)$.

In view of a future investigation on commercial milk at the dairy company, preliminary results showed quantifiable BPA levels at all stages of the dairy chain, albeit below the specific migration limit value. One feature of BPA pollution is its ubiquity, which is directly related to anthropogenic activities (Georgescu and Georgescu, 2013). The environmental half-life results in a continual increase of the global concentration in air, soil, and water. Consequently, BPA can enter plants and animals at the bottom of the food chain, and exposure of the cow to environmental sources may induce excretion of BPA through the milk (Carnevali et al., 2017). Environmental contamination of the geographic area where the milk was produced, even at very low levels, may have played a role in milk chain contamination. Mechanical milking may also relate to raw milk contamination (Chege and Ndungu, 2016), but considering the low concentrations detected in mechanically obtained milk, we hypothesized that no noticeable migration from materials of the milking machine into raw milk occurred. According to Chege and Ndungu (2016), we concluded that storage of milk in the cooling tank played a more important role as a contamination source on the farm, through migration of BPA from contact materials. We found that BPA residues tended to accumulate at the end of the milk chain, and the variability in BPA concentration along the milk chain may be partly attributed to the sampling method or different contamination levels of milk from the cooling tank obtained from the pool of all cows present on the farm.

We are not aware of any similar study on BPA in raw cow milk. Most studies have reported BPA levels only in commercial milk (from 14.0 to $521.0 \mu \mathrm{g} / \mathrm{L}$ ) or cheese $(2.24 \mu \mathrm{g} / \mathrm{kg}$; Grumetto et al., 2013; Wlodarczyk, 2015; Mercogliano and Santonicola, 2018). If we compare our data with contamination levels of commercial milk, the latter are generally higher. These differences between raw and commercial milk could be related to different factors, such as the quality of the materials in contact with milk during manufacturing and the treatment conditions at the dairy company (e.g., thermal treatments, packaging; Grumetto et al., 2013). The BPA concentrations quantified in raw milk were high compared with those in principal foodstuffs of animal origin, such as meat $(0.48 \mu \mathrm{g} / \mathrm{kg})$, poultry liver pate $(0.48 \mu \mathrm{g} / \mathrm{kg})$, and butter $(0.56 \mu \mathrm{g} / \mathrm{kg})$, but lower than those detected in sea fish $(0.89 \mu \mathrm{g} / \mathrm{kg})$, eggs $(<2-2.4 \mu \mathrm{g} / \mathrm{kg})$, and fats $(<4 \mu \mathrm{g} / \mathrm{kg}$; Wlodarczyk, 2015).

The presence of contaminants in raw milk is a key issue for milk safety due to the risk of direct toxic effects on consumers. Our results confirmed that BPA contamination, even at low levels, in a widely consumed

Table 3. Bisphenol A (BPA) daily intakes $(\mu \mathrm{g} / \mathrm{kg}$ of BW per day) through consumption of milk collected at the farm and cheese consumption

\begin{tabular}{|c|c|c|c|c|c|c|}
\hline Age (yr) & \multicolumn{3}{|c|}{ Daily intake through milk consumption ${ }^{1}$} & \multicolumn{3}{|c|}{ Daily intake through cheese consumption ${ }^{2}$} \\
\hline $3-10$ & 0.0065 & 0.0050 & 0.0068 & 0.0072 & 0.005 & 0.0076 \\
\hline $10-18$ & 0.0029 & 0.0022 & 0.0031 & 0.0049 & 0.0038 & 0.0052 \\
\hline $18-65$ & 0.0015 & 0.0011 & 0.0015 & 0.0033 & 0.0025 & 0.0035 \\
\hline
\end{tabular}

${ }^{1}$ Calculated based on average milk consumption and BPA concentrations, the latter relative to the samples obtained manually, mechanically, or from the cooling tank.

${ }^{2}$ Calculated based on average cheese consumption and BPA concentrations calculated considering that $1 \mathrm{~kg}$ of cheese requires about $5 \mathrm{~L}$ of milk. The BPA concentrations in milk are relative to sample units obtained manually, mechanically, or from the cooling tank. 
food such as milk represents a food safety concern (Kolatorova et al., 2017). The food chain approach and the application of monitoring programs along the dairy supply chain may represent a perspective to ensure food safety.

In terms of human exposure to BPA, diet as the main source and age of the consumer are the most important factors, particularly when exposure through milk and dairy products is considered (EFSA, 2015). A study on the dietary intake assessment of Italian infants (age 0-4 mo) considered 2 possible scenarios: a medium case (median concentrations of contaminants and infants who consume a medium quantity of milk daily) and a worst case (maximum concentrations of contaminants, and infants who consume a higher quantity of milk daily). In both cases, the highest intake occurred on d 30 of life because the amount of consumed milk starts increasing when the baby's weight is still low (Cirillo et al., 2015). With the introduction of common foods into the diet, a further increase of BPA exposure was observed for 0- to 6-mo-old infants (0.062-0.078 $\mu \mathrm{g} / \mathrm{kg}$ of BW per day) and 12- to 36-mo-old children $(0.156-0.182 \mu \mathrm{g} / \mathrm{kg}$ of BW per day). In contrast, for older children and adolescents (3-17 yr old), exposure decreased with age $(0.05-0.06 \mu \mathrm{g} / \mathrm{kg}$ of BW per day), probably due to the increase in BW relative to food consumption. For adults (18 yr and over), mean dietary exposure ranged from 0.038 to $0.040 \mu \mathrm{g} / \mathrm{kg}$ of BW per day (Bemrah et al., 2014). Slightly higher levels of exposure were reported by EFSA for infants $(0.857 \mu \mathrm{g} / \mathrm{kg}$ of BW per day), adolescents ( $\mu \mathrm{g} / \mathrm{kg}$ of BW per day), and adults $(0.388 \mu \mathrm{g} / \mathrm{kg}$ of BW per day; EFSA, 2015).

In our study, BPA levels of exposure along the milk chain were below the t-TDI value for all age groups of consumers, according to bibliographic data (Bemrah et al., 2014; EFSA, 2015). We observed that (1) the overall BPA intake of a typical Italian consumer might be higher than reported values because of other contaminated food items; (2) a synergistic effect of BPA analogs and other pollutants may occur; and (3) BPA may induce adverse effects and influence human exposure also at low levels (EFSA, 2015).

In conclusion, the age at which an organism is exposed to an endocrine disruptor can be crucial, because although very low doses can have lasting effects during human development, higher levels are needed to produce harmful effects in adults (EFSA, 2015; Kolatorova et al., 2017). Considering the nature of the potential adverse effects, further precautionary steps should be taken in vulnerable population groups, and a specific tolerable daily intake for infants should be recommended (Cirillo et al., 2015; Mercogliano and Santonicola 2018). In our study, the detected concentrations of BPA contamination, although low, might influence the contamination levels of commercial milk at the dairy company. Therefore, the proposed contamination pathways should be further explored, according to a new model of risk assessment based on identifying hazards at each stage of the milk chain.

\section{REFERENCES}

Bansal, A., J. Henao-Mejia, and R. A. Simmons. 2018. Immune system: An emerging player in mediating effects of endocrine disruptors on metabolic health. Endocrinology 159:32-45.

Beghini, G. 1981. L'alimentazione del bambino in età scolare. R. Bulgarelli, ed. Cortina, Verona, Italy.

Bemrah, N., J. Jean, G. Rivière, M. Sanaa, S. Leconte, M. Bachelot, Y. Deceuninck, B. Le Bizec, X. Dauchy, A. C. Roudot, V. Camel, K. Grob, C. Feidt, N. Picard-Hagen, P. M. Badot, F. Foures, and J. C. Leblanc. 2014. Assessment of dietary exposure to bisphenol A in the French population with a special focus on risk characterization for pregnant French women. Food Chem. Toxicol. 72:90-97.

Carnevali, O., V. Notarstefano, I. Olivotto, M. Graziano, P. Gallo, I Di Marco Pisciottano, L. Vaccari, A. Mandich, E. Giorgini, and F. Maradonna. 2017. Dietary administration of EDC mixtures: A focus on fish lipid metabolism. Aquat. Toxicol. 185:95-104.

Chege, P., and Z. Ndungu. 2016. Chapter 1. Analysis of contamination points of milk through the whole value chain process and the quality of milk products. Pages 1-14 in the Dairy Industry Food-Avid Science. Mahavir House, Himayathnagar Hyderabad, Telangana, India.

Cirillo, T., G. Latini, M. A. Castaldi, L. Dipaola, E. Fasano, F. Esposito, G. Scognamiglio, F. Di Francesco, and L. Cobellis. 2015 Exposure to di-2-ethylhexyl phthalate, di-n-butyl phthalate and bisphenol A through infant formulas. J. Agric. Food Chem. 63:3303-3310.

EFSA (European Food Safety Authority). 2015. Scientific Opinion on the risks to public health related to the presence of bisphenol A (BPA) in foodstuffs: Executive summary. EFSA J. 13:3978. https: //doi.org/10.2903/j.efsa.2015.3978.

European Union. 2018. Regulation (EU) No 2018/213 of 12 February 2018 on the use of bisphenol A in varnishes and coatings intended to come into contact with food and amending Regulation (EU) No $10 / 2011$ as regards the use of that substance in plastic food contact materials. European Union, Brussels, Belgium.

Galloway, T. S. 2015. Micro- and nano-plastics and human health Pages 343-366 in Marine Anthropogenic Litter. M. Bergmann, L. Gutow, and M. Klages, ed. Springer International, New York, NY.

Georgescu, B., and C. E. Georgescu. 2013. Bisphenol A levels in commercial milk, infant formula and dairy products. Anim. Biol Anim. Husb. 5:171-174.

Grumetto, L., O. Gennari, D. Montesano, R. Ferracane, A. Ritieni, S. Albrizio, and F. Barbato. 2013. Determination of five bisphenols in commercial milk samples by liquid chromatography coupled to fluorescence detection. J. Food Prot. 76:1590-1596.

Ho, K. L., K. K. Yuen, M. S. Yau, M. B. Murphy, Y. Wan, B. M. W. Fong, S. Tam, J. P. Giesy, K. S. Y. Leung, and M. H. W. Lam. 2017. Glucuronide and sulfate conjugates of bisphenol A: Chemical synthesis and correlation between their urinary levels and plasma bisphenol A content in voluntary human donors. Arch. Environ. Contam. Toxicol. 73:410-420.

Kolatorova, L., M. Duskova, J. Vitku, and L. Starka. 2017. Prenatal exposure to Bisphenols and Parabens and impacts on human physiology. Physiol. Res. 66:S305-S315.

Leclercq, C., D. Arcella, R. Piccinelli, S. Sette, and C. Le Donne. 2009. The Italian National Food Consumption Survey INRANSCAI 2005-06: Main results in terms of food consumption. Public Health Nutr. 12:2504-2532. 
Maradonna, F., V. Nozzi, L. Dalla Valle, I. Traversi, G. Gioacchini, F. Benato, E. Coletti, P. Gallo, I. Di Marco Piscioottano, D. G. Mita, G. Hardiman, A. Mandich, and O. Carnevali. 2014. A developmental hepatotoxicity study of dietary bisphenol A in Sparus aurata juveniles. Comp. Biochem. Physiol. C Toxicol. Pharmacol. 166:1-13.

Mercogliano, R., and S. Santonicola. 2018. Investigation on bisphenol A levels in human milk and dairy supply chain: A review. Food Chem. Toxicol. 114:98-107.

Nachman, R. M., J. C. Hartle, P. S. Lees, and J. D. Groopman. 2014. Early life metabolism of bisphenol A: A systematic review of the literature. Curr. Environ. Health Rep. 1:90-100.

Quesnot, N., S. Bucher, B. Fromenty, and M. A. Robin. 2014. Modulation of metabolizing enzymes by bisphenol $\mathrm{A}$ in human and animal models. Chem. Res. Toxicol. 27:1463-1473.

Santonicola, S., A. De Felice, L. Cobellis, N. Passariello, A. Peluso, N. Murru, M. C. Ferrante, and R. Mercogliano. 2017. Comparative study on the occurrence of polycyclic aromatic hydrocarbons in breast milk and infant formula and risk assessment. Chemosphere 175:383-390.

van Asselt, E. D., H. J. der Fels-Klerx, H. J. P. Marvin, H. Bokhorstvan de Veen, and M. N. Groot. 2017. Overview of food safety hazards in the European dairy supply chain. Compr. Rev. Food Sci. Food Saf. 16:59-75.

Watabe, Y., T. Kondo, H. Imai, M. Morita, N. Tanaka, and K. Hosoya. 2004. Reducing bisphenol A contamination from analytical procedures to determine ultralow levels in environmental samples using automated HPLC microanalysis. Anal. Chem. 76:105-109.

Wlodarczyk, E. 2015. Occurrence of bisphenol A and its effects on the human body. Arch. Physiother. Glob. Res. 19:13-26.

Ye, X., X. Zhou, R. Hennings, J. Kramer, and A. M. Calafat. 2013. Potential external contamination with bisphenol A and other ubiquitous organic environmental chemicals during biomonitoring analysis: an elusive laboratory challenge. Environ. Health Perspect. 121:283-286. 